\title{
Aplicação do produto de evapotranspiração do MODIS para uma área de pastagem na Amazônia ocidental
}

Application of MODIS evapotranspiration product for a pasture area in the Western Amazon

\author{
Agni Cristina Carvalho de Brito ${ }^{1}$, Nara Luisa Reis de Andrade ${ }^{2}$, Larissa Santos Fambri², Camila \\ Bermond Ruezzene ${ }^{2}$ e Renata Gonçalves Aguiar ${ }^{2}$
}

${ }^{1}$ UNESC, Cacoal, Brasil

${ }^{2}$ Universidade Federal de Rondônia - UNIR, Ji-Paraná, Brasil

\begin{abstract}
Resumo
Os processos de uso e ocupação da terra geram intervenções nos ecossistemas naturais tornando-os susceptíveis a reações, das quais se destacam as alterações nos processos que regem a ciclagem da água, ressaltando a importância de se monitorar o comportamento da evapotranspiração. Nesse sentido, o objetivo do presente estudo foi verificar a aplicabilidade do produto de evapotranspiração originada pelo sensor MODIS para uma área de pastagem, na Fazenda Nossa Senhora no municipio de Ouro Preto do Oeste - Rondônia. Foram utilizados dados de evapotranspiração do sensor MODIS (Terra/Aqua), estimados pelo algoritmo MOD16, e de torre micrometeorológica situada em área de pastagem, medidos pelo sistema eddy covariance, no periodo de 2003 a 2010. Verificou-se que para o conjunto de dados ET Eddy x ET MOD16 (Controle de qualidade - QC 0/8), os dados de ET MOD16 (QC 0/8) superestimam a evapotranspiração, quando comparados ao ET Eddy, e apresentam uma maior amplitude. Não foi identificada uma correlação linear entre os conjuntos de dados do estudo, no entanto, as variações sazonais são captadas pelo produto, apresentando boa aproximação com os dados do ET Eddy, especialmente nos periodos de transição.
\end{abstract}

Palavras-chave: Sensoriamento remoto. ET MOD16. Fluxo de calor latente

Abstract

The processes of land use and occupation generate interventions in the natural ecosystems making them susceptible to reactions, such as changes in the processes that govern water cycling, emphasizing the importance of monitoring the evapotranspiration behavior. In this sense, the objective of this study was to verify the applicability of the evaporation product originated by the MODIS sensor to a pasture area, from 2003 to 2010, at Fazenda Nossa Senhora in the municipality of Ouro Preto do Oeste - Rondonia. Were used evapotranspiration data from the MODIS (Terra / Aqua) sensor, estimated by MOD16 algorithm, and micrometeorological tower located in the pasture area, generated by eddy covariance system. It was verified that for ET Eddy x ET MOD16 (Quality control - QC 0/8) data set, ET MOD16 (QC 0/8) data showed evapotranspiration values above those of ET Eddy and with a greater amplitude. A linear correlation between the study datasets was not identified, however, seasonal variations are captured by product, showing good approximation with ET Eddy data, especially in the transition periods.

Keywords: Remote sensing. ET MOD16. Latent heat flux 


\section{Introdução}

Os recursos naturais utilizados pela humanidade dispõem-se em quantidade e qualidade condicional às condições do sistema que os contém. Com a intensificação do uso da terra e pelo fato da vegetação ser considerada influente nas variações de dinâmica da atmosfera e condições de superfície (ARTAXO et al., 2014), torna-se fundamental o emprego de estudos com o intuito de compreender as interações de variáveis dominantes que regem as trocas de massa e energia e entre a superfície e a atmosfera, destacando-se a importância de se monitorar o comportamento da evapotranspiração, pois, a diminuição das áreas florestadas, modifica a dinâmica da evapotranspiração e a entrada de energia (von RANDOW et al., 2004).

Devido à insuficiente disponibilidade de dados meteorológicos, destaca-se o sensoriamento remoto, devido à facilidade da coleta dos dados e por fornecer informações valiosas para a avaliação das condições da vegetação, auxiliando em tomadas de decisões que visam a melhoria dos sistemas produtivos locais (ANDRADE et al., 2015).

O sensoriamento remoto permite a estimativa da evapotranspiração em grandes áreas, como função das características biofísicas encontradas em cada pixel, com a vantagem do emprego de imagens de satélites na estimativa da evapotranspiração, em escala regional, sem a necessidade de se quantificarem outros parâmetros hidrológicos, como a umidade do solo (LIMA et al., 2014).

Com a intensificação do uso da terra e suas consequências no clima regional e global, é imprescindível o estudo dos processos de funcionamento do ecossistema amazônico com diferentes tipos de cobertura. Dessarte, o presente estudo teve como objetivo, verificar a aplicabilidade do produto evapotranspiração originado pelo sensor MODIS para uma área de pastagem, no período de 2003 a 2010, com base em uma série de oito anos de dados.

\section{Metodologia}

\section{1. Área de estudo e aquisição dos dados}

A pesquisa foi realizada em um dos sítios experimentais pertencentes ao Programa de Grande Escala da Biosfera-Atmosfera na Amazônia (LBA), na Fazenda Nossa Senhora (FNS), no município de Ouro Preto do Oeste, sob as coordenadas $10^{\circ}$ $45^{\prime} 44^{\prime}$ 'S / $62^{\circ} 21^{\prime} 27^{\prime \prime}$ O. A cobertura vegetal predominante é a gramínea Brachiaria brizantha, além de pequenas palmeiras dispersas. O solo é classificado como podzólico vermelho-amarelo (HODNETT et al., 1996).

A delimitação da área de estudo deu-se em âmbito de microbacia hidrográfica e com uma área de aproximadamente 14,89 $\mathrm{km}^{2}$, as quais foram geradas a partir de dados numéricos de relevo e da topografia do Brasil, obtidos por satélite durante a missão SRTM, disponibilizados no sítio da EMBRAPA (MIRANDA, 2017).

Os dados obtidos para verificar a aplicabilidade do produto de evapotranspiração originadas pelo sensor MODIS para uma área de pastagem, estão dispostos na Tabela 1 .

Tabela 1 - Descrição das variáveis fluxo de calor latente (LE), temperatura do ar (T ar) e evapotranspiração (ET) referentes ao entorno da torre micrometeorológica situada na Fazenda Nossa Senhora (FNS) e respectivas fontes de dados

\begin{tabular}{c|c|c|c}
\hline Variável & Unidade & Intervalo de dados disponíveis & Fonte \\
\hline LE & $\mathrm{W} \mathrm{m}^{-2}$ & 2003 a $2010(*$ ex. 2007) & \multirow{2}{*}{ Torre micrometeorológica } \\
\hline T ar & ${ }^{\circ} \mathrm{C}$ & 2003 a 2010 & \\
\hline ET MOD16 & $\mathrm{mm}$ & 2003 a 2010 & Sensor MODIS - Terra/Aqua \\
\hline
\end{tabular}

Nota: *não houve registro de fluxo de calor latente (LE) no ano de 2007

O fluxo calor latente (LE) foi medido por um sistema de alta frequência de superfície, presente na torre micrometeorológica. O equipamento usado foi um anemômetro sônico tridimensional (Solent 1012R2, Gill Instruments, Lymington, UK); e um analisador de gás por infravermelho de caminho aberto (IRGA, Li-7500, LICOR Inc., Lincoln USA), instalados a quatro metros da superfície, e conectados a um microcomputador tipo "palmtop" que efetua a leitura dos sensores em uma frequência de 10,4 Hz. Os dados brutos registrados pelos sensores são processados em um computador através do software Alteddy (ELBERS, 1998), gerando dados de fluxos de energia, por meio do sistema de covariância dos vórtices turbulentos (eddy covariance).

Encontra-se também instalado na torre um termohigrômetro (HMP35A e HMP45C, Vaisala Inc., FIN) a dez metros de altura, o qual registra medidas de $\mathrm{T}$ ar a cada $30 \mathrm{~s}$ e realiza médias a cada $10 \mathrm{~min}$, sendo essas armazenadas em um datalogger (CR10X, Campbell Scientific Instrument, Utah, USA).

Por sua vez, a evapotranspiração (ET MOD16) foi gerada a partir do registro de informações pelo sensor MODIS dos satélites Aqua/Terra e obtida através do sítio Oak Ridge National Laboratory Distributed Active Archive Center for Biogeochemical Dynamics (ORNL DAAC). Os dados de evapotranspiração do produto MOD16 (ET MOD16) são fornecidos por pixels, com 
resolução espacial de aproximadamente $1 \mathrm{~km}^{2}$ e resolução temporal de oito dias (dias Julianos). Sendo assim, a série de ET MOD16 foi adquirida para uma área de aproximadamente $3 \times 3 \mathrm{~km}$, onde o pixel central corresponde a localização da torre micrometeorológica na FNS.

\subsection{Estimativa de evapotranspiração}

Para obtenção dos valores de evapotranspiração, foram utilizados os dados de fluxo de LE e T ar, sendo que após a retirada dos dados inválidos, efetuou-se o pareamento dos mesmos. A organização das variáveis foi realizada de acordo com os intervalos de 30 minutos dos dados de LE. Em seguida aplicou-se as Equações 1a e 1b para conversão dos dados de LE (W m²) em evapotranspiração horária, ETh $\left(\mathrm{mm} \mathrm{h}^{-1}\right)$, com os intervalos de 30 minutos.

$$
\begin{aligned}
& \lambda=[2,501-0,00236(\operatorname{Tar}-273,15)] 10^{6} \\
& \mathrm{ETh}=\left(\lambda \mathrm{ET}^{*} 3600\right) / \lambda
\end{aligned}
$$

Em que $\lambda$ representa o calor latente de vaporização da água, o ETh é a evapotranspiração horária e $\lambda \mathrm{ET}\left(\mathrm{W} \mathrm{m}^{-2}\right)$ refere-se ao fluxo de calor latente (LE).

Para fins de comparação, foi utilizado o produto de sensoriamento remoto MOD16 de evapotranspiração global. Esse possui resolução espacial de aproximadamente $1 \mathrm{~km}^{2}$, e seu algoritmo MOD16 usa como entrada dados ópticos e dados meteorológicos de reanálise. Os dados ópticos de entrada para cálculo do produto MOD16 consistem em: classificação de uso e cobertura da terra do produto MOD12Q1; índice de área foliar (IAF) e fração de radiação fotossinteticamente ativa (fPAR) do produto MOD15A2; e albedo do produto MCD43A.

O controle de qualidade (QC) é gerado pelo produto MOD16A2QC e foi efetuado conforme descrito por Andrade (2013). Esse produto foi utilizado neste estudo como parâmetro de filtragem dos dados de ET. A fim de definir quais códigos arbitram em um melhor desempenho na eliminação de valores inapropriados de ET, deliberou-se o uso dos dados do MOD16 com arranjos até o código 16, formando quatro tipos de conjunto de dados: um considerando apenas os dados correspondentes ao QC 0, controle de qualidade mais restritivo; outro considerando os dados referentes aos QCs 0 e 8; outro considerando os dados atribuídos aos QCs 0, 8 e 16; e por último usando todos os valores de ET (sem filtragem).

Para todos os produtos MOD16 trabalhados, foram aplicadas transformações nos valores de ET para milímetros (mm), conforme descrevem Mu, Zhao e Running (2011).

\subsection{Análises estatísticas}

A título de identificação da aplicabilidade da série de dados de evapotranspiração do sensor MODIS, foram empregadas para todas as quatro classes de dados do produto MOD16, de acordo com o QC, em pareamento com a série de dados de medida gerados pela covariância dos vórtices turbulentos (ET Eddy), análises estatísticas de: correlação de Pearson (r), a um nível de significância de 0,05; raiz do erro médio quadrático (RMSE) e percentual de diferença entre as médias.

\section{Resultados e Discussão}

\subsection{Aproveitamento de dados de evapotranspiração da estação micrometeorológica}

Após a retirada dos dados espúrios, contabilizou-se a porcentagem de aproveitamento dos mesmos. Todos os anos em estudo (2003 a 2010, exceto 2007), apresentaram baixas porcentagens de aproveitamento, variando entre $12,33 \%$ a $47,67 \%$ o percentual diário de ET Eddy, havendo um pequeno acréscimo de aproveitamento, para a maioria dos anos, quando computados os valores de ET Eddy médias de 8 dias. Foi necessária a adequação dos dados em médias de 8 dias para avaliação dos dados do produto MOD16, a fim de que os valores da série de ET Eddy fossem ajustados na mesma resolução temporal da série de ET MOD16.

As falhas podem ser atribuídas a problemas nos sensores, na manutenção técnica ou erros de coleta e armazenamento, comum na execução de medidas contínuas realizadas por torres micrometeorológicas, podendo variar, para diferentes áreas estudadas na região Amazônica, em média entre 0,3 e 59\% (IMBUZEIRO, 2005).

\subsection{Avaliação da estimativa de evapotranspiração do produto MOD16}

A comparação estatística entre os pares de dados de evapotranspiração gerados pelo sistema de covariância dos vórtices turbulentos (ET Eddy) e produtos do MOD16 podem ser observados na Tabela 2. 
Tabela 2 - Conjunto de dados de evapotranspiração gerados pelo sistema de covariância dos vórtices turbulentos (ET Eddy) e produtos do MOD16 de quatro formas de arranjo de séries de dados, considerando controle de qualidade (QC), referente ao entorno da torre micrometeorológica situada na Fazenda Nossa Senhora (FNS), quantidade de dados (n), análises estatísticas de correlação de Pearson ( $\mathrm{r})(\mathrm{p}<0,05)$, percentual de diferença entre as médias e raiz do erro médio quadrático (RMSE).

\begin{tabular}{c|c|c|c|c}
\hline Conjunto de dados & $\mathrm{n}$ & $\mathrm{r}(\mathrm{p}<0,05)$ & $\begin{array}{c}\text { Diferença entre as médias } \\
(\%)\end{array}$ & $\begin{array}{c}\text { RMSE } \\
\left(\mathrm{mm} \mathrm{dia}^{-1}\right)\end{array}$ \\
\hline ET Eddy x ${ }^{1}$ ET MOD16 $(0)$ & 67 & $0,044(0,050)$ & 1,973 & 1,630 \\
\hline ET Eddy ${ }^{2}$ ET MOD16 $(0 / 8)$ & 98 & $0,086(0,092)$ & $-7,844$ & 1,592 \\
\hline ET Eddy ${ }^{3}$ ET MO16 $(0 / 8 / 16)$ & 102 & $0,078(0,082)$ & $-9,463$ & 1,597 \\
\hline ET Eddy ${ }^{4}$ ET MOD16 (T) & 119 & $0,009(0,010)$ & $-16,265$ & 1,667 \\
\hline
\end{tabular}

Nota: ${ }^{1}$ ET MOD16 (0) considera apenas os dados correspondentes ao QC 0 (controle de qualidade mais restritivo); ${ }^{2}$ ET MOD16 (0/8) considera os dados referentes aos QCs 0 e 8; ${ }^{3}$ ET MOD16 (0/8/16) considera os dados atribuídos aos QCs 0, 8 e 16; e "ET MOD16 (T) usa todos os valores de ET, sem considerar controle de qualidade.

Em geral, as análises estatísticas empregadas indicaram não concordância entre os pares de dados, fato perceptível em função dos coeficientes de Pearson que apresentaram valores muito próximos ao zero.

A série ET MOD16 (0/8) foi a que apresentou melhor r quando comparado com os dados da série de ET Eddy, assim como apresentou o menor RMSE. Apesar de que a melhor aproximação entre as médias foi apresentada no pareamento da série ET Eddy com a série ET MOD16 (0), considerou-se de melhor desempenho os pares de dados de ET Eddy com a sequência de ET MOD16 (0/8).

Kim et al. (2012), analisando séries de dados na Ásia nos anos de 2000 a 2006, das 17 localidades estudadas, duas foram descritas com cobertura vegetal por pastagem, cujos dados também não apresentaram correlação significativa, com r de 0,12 e 0,18 e RMSE de 5,76 e 7,66 .

Verifica-se com essas informações que em geral, para o conjunto de dados ET Eddy x ET MOD16 (0/8), os dados de ET MOD16 $(0 / 8)$ apresentaram valores de evapotranspiração acima dos de ET Eddy e com uma maior amplitude para as localidades dos sítios situados na FNS e REBIO Jaru da região sudoeste da Amazônia. Tal fato pode ser devido à resolução temporal do sensor MODIS (8 dias), que não permite a captura das oscilações menores (horárias, diárias) dos processos evapotranspirativos. Bem como, pode se implicar como possível fator o elevado número de falhas nos dados do sistema eddy covariance, ressaltando sua sensibilidade a situações extremas do tempo, por exemplo, na época chuvosa (ANDRADE, 2013; RUHOFF, 2011).

De acordo com as médias estacionais de ET Eddy e ET MOD16 (Figura 1), o valor mínimo para ET Eddy foi de 2,39 $\mathrm{mm} \mathrm{d}^{-1}$ (estação seca) e máximo de 3,06 $\mathrm{mm} \mathrm{d}^{-1}$ (período seco-úmido). Para ET MOD16 o valor mínimo de evapotranspiração foi de 1,35 $\mathrm{mm} \mathrm{d}^{-1}$ (estação seca) e máximo de 4,15 $\mathrm{mm} \mathrm{d}^{-1}$ (estação úmida).

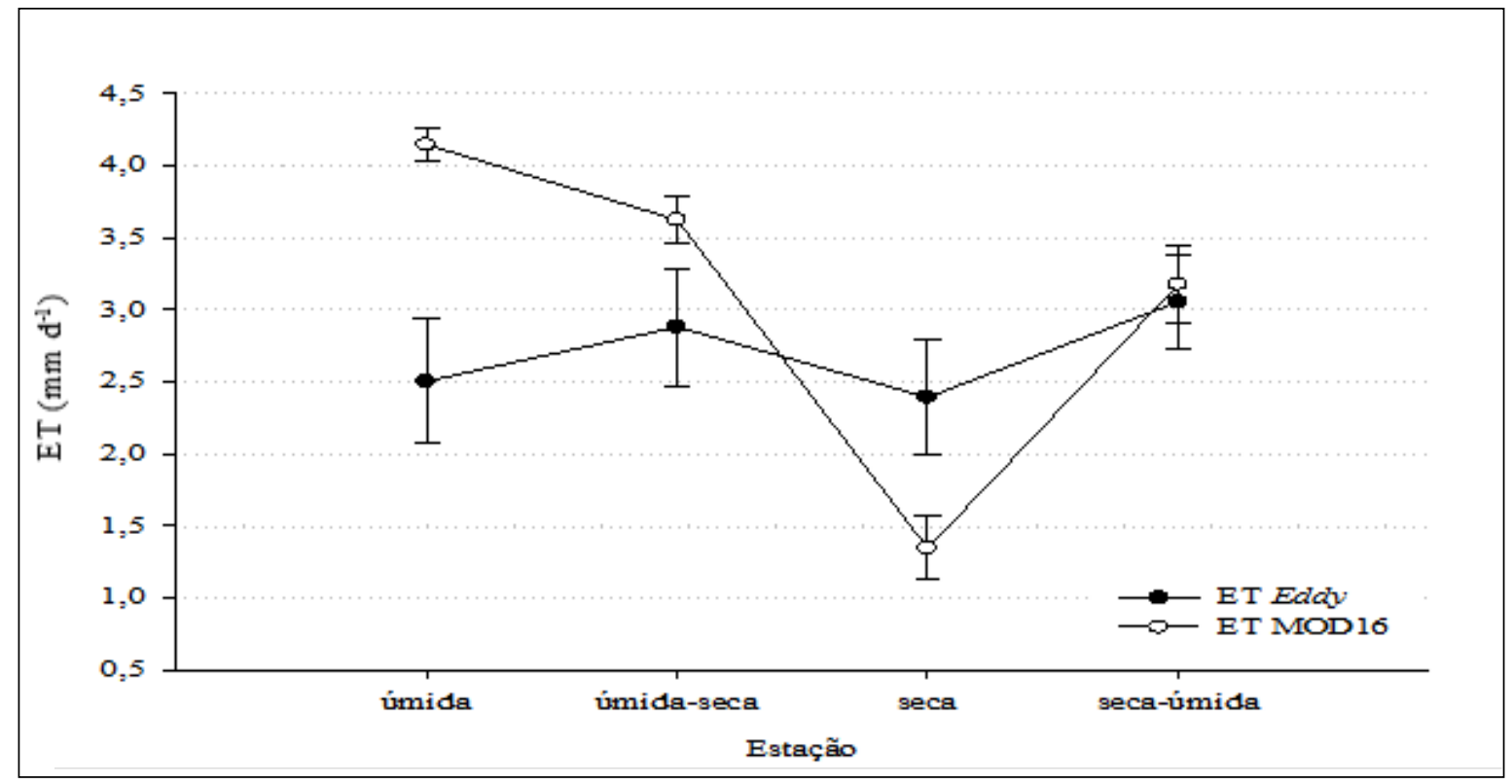

Figura 1 - Médias de evapotranspiração (intervalo de confiança de 95\%) nos períodos úmido, úmido-seco, seco e seco-úmido gerados pela covariância dos vórtices turbulentos (ET Eddy) e pelo produto MOD16 (ET MOD16) para as séries de dados dos anos de 2003 a 2010, exceto 2007, referente ao entorno da torre micrometeorológica situada na Fazenda Nossa Senhora (FNS). 
O comportamento da evapotranspiração observado por estudos na região Amazônica demonstra que em geral na estação úmida, apesar da grande disponibilidade de água, os valores de ET não são maiores por haver uma redução de energia para agir no sistema, devido à interceptação da radiação pela cobertura de nuvens típica dessa estação (COSTA et al., 2010).

Sendo assim, normalmente as faixas de valores máximos de ET encontram-se nos períodos de transição úmida-seca e seca-úmida onde a disponibilidade de água nos elementos da superfície não reduz tanto quanto na estação seca e a superfície não se encontra coberta pelas nuvens tanto quanto na estação úmida. Esse comportamento é claramente notado pelas médias estacionais de ET Eddy.

Comparativamente, também é durante os períodos de transição que ET Eddy e ET MOD16 apresentam maior aproximação, podendo-se sugerir que tal fato se deve à maior estabilidade das condições do tempo nesse período, bem como, a menor influência da cobertura de nuvens e presença de aerossóis.

No entanto, no comportamento dos valores de ET MOD16, nota-se que em especial na estação úmida, o modelo superestima a média de ET Eddy observada a campo mais do que nos demais períodos. Os estudos de Ruhoff (2011) e Andrade (2013) apontam como justificativa a maior cobertura de nuvens da superfície nesse período de alta pluviosidade associado ao fato de que os produtos do sensor MODIS usam fundamentalmente como dados de entrada parâmetros da cobertura da terra. Pode ocorrer ainda, devido a menor eficiência do sistema eddy covariance na presença de água, ocasionando falhas nas medições em período de elevada pluviosidade.

\section{Conclusões}

Não foi identificada uma correlação linear entre os conjuntos de dados em questão, entretanto, análises de variações mensais e de médias indicam uma boa aproximação entre os conjuntos de dados estimados pelo algoritmo MOD16 e pelo sistema eddy covariance, especialmente nos períodos de transição.

Ressalta-se que a evapotranspiração é uma variável hidrológica fundamental, por isso a importância da realização de estudos detalhados para uma melhor compreensão de suas variações espaço-temporais.

\section{Agradecimentos}

As autoras agradecem ao Programa de Grande Escala da Biosfera-Atmosfera na Amazônia - LBA e à Universidade Federal de Rondônia - UNIR.

\section{Referências}

ANDRADE NLR, CORILAÇO LPF, SANCHES L, AGUIAR RG, ROCHA DRS, BARBOSA HA. Aplicabilidade do produto de evapotranspiração do Land-SAF em uma área de floresta na Amazônia Ocidental. In: Anais do $8^{\circ}$ Simpósio Brasileiro de Sensoriamento Remoto (Internet); 2015, abril 25-29; João Pessoa, Brasil, 2015 (cited 2015 jan 04). Available from: http://www.dsr.inpe.br/sbsr2015/files/p0790.pdf.

ANDRADE NLR. Fluxo de calor latente em uma floresta tropical da Amazônia: análise de séries temporais com wavelets e do produto MOD16 [thesis]. Cuiabá: Instituto de Física/UFMT; 2013. 37 p.

ARTAXO P, DIAS MAFS, NAGY L, LUIZÃO FJ, CUNHA HB, QUESADA CAN, et al. Perspectivas de pesquisas na relação entre clima e o funcionamento da floresta amazônica. Ciencia Cultura. 2014;66(3);41-46.

COSTA MH, BIAJOLI MC, SANCHES L, MALHADO ACM, HUTYRA LR, ROCHA, H, et al. [Atmospheric versus vegetation controls of Amazonian tropical rain forest evapotranspiration: Are the wet and seasonally dry rain forests any different?] Journal of Geophysical Research. 2010;115;1-9. Inglese.

ELBERS, JA. Eddy correlation system: user manual version 2.0. Alterra, Wageningen, The Netherlands. $1988 ; 39$.

IMBUZEIRO, HMA. Calibração de um modelo para simular fluxos de massa e energia entre a floresta amazônica e a atmosfera usando múltiplos sítios [dissertation]. Viçosa: Mestrado em Meteorologia Agrícola/UFV; 2005.9 p.

KIM HW, HWANG K, MU Q, LEE SO, CHOI M. [Validation of MODIS 16 Global Terrestrial Evapotranspiration Products in Various Climates and Land Cover Types in Asia]. KSCE Journal of Civil Engineering. 2012;16(2);229238. Inglese. 
LIMA EP, SEDIYAMA GC, ANDRADE RG, LOPES VD, SILVA BB. Evapotranspiração Real Diária em Sub-bacias do Paracatu, Utilizando Produtos do Sensor Modis1. Revista Ceres. 2014;61(1);17-27.

MIRANDA EE. Brasil em Relevo. Campinas: Embrapa Monitoramento por Satélite. 2005 [cited 2018 jan 04]. Available from: http://www.relevobr.cnpm.embrapa.br.

MU Q, ZHAO M, RUNNING SW. [Improvements to a MODIS global terrestrial evapotranspiration algorithm. Remote Sensing of Environment]. 2011;115(2);1781-1800. Inglese.

ORNL DAAC. Available from: http://daac.ornl.gov/. Acesso em: 7 Set. 2017.

RUHOFF AL. Sensoriamento remoto aplicado à estimativa da evapotranspiração em biomas tropicais [thesis]. Porto alegre: Doutorado em Recursos Hídricos e Saneamento Ambiental/UFRGS, 2011.

Von RANDOW C, MANZI AO, KRUIJT B, OLIVEIRA PJ, ZANCHI F, SILVA RL, et al. [Comparative measurements and seasonal variations in energy and carbon Exchange over forest and pasture in South West Amazonia]. Theoretical and Applied Climatology. 2004;78(1);5-26. Inglese.

Agni Cristina Carvalho de Brito

UNESC, Cacoal, Brasil

E-mail: agnibrito@gmail.com

Nara Luisa Reis de Andrade

Universidade Federal de Rondônia - UNIR, Ji-Paraná, Brasil E-mail: naraluisar@gmail.com

Larissa Santos Fambri

Universidade Federal de Rondônia - UNIR, Ji-Paraná, Brasil E-mail: larissasfambri@gmail.com

Camila Bermond Ruezzene

Universidade Federal de Rondônia - UNIR, Ji-Paraná, Brasil E-mail: camilaruezzene@gmail.com

Renata Gonçalves Aguiar

Universidade Federal de Rondônia - UNIR, Ji-Paraná, Brasil E-mail: rgaguiar@unir.br 